\title{
Eco-friendly innovative gripping systems actuated by pneumatic muscles
}

\author{
Andrea Deaconescu and Tudor Deaconescu \\ Industrial Engineering and Management Department, Transilvania University, \\ 29, Eroilor Bd., Brasov, 500036, Romania.
}

\begin{abstract}
The paper focuses on the development of innovative, bionic, eco-friendly and light gripping systems. Within this broader context the paper presents and discusses four innovative constructive solutions for a gripping system mountable on an industrial robot, based on actuation by a linear pneumatic muscle and transmission of motion by gear and rack mechanism. The method underlying the development of these novel gripping systems is the creation of analogy based concepts, the models being inspired and adopted from nature.
\end{abstract}

Keywords: Gripper systems, Pnaumatic muscle, Compliance

\section{INTRODUCTION}

The most important function of the human hand is gripping, defined as the "action of gripping, of seizing by means of fingers, claws, of tweezers, etc." [1]. While the capacity of gripping by means of the extremities of the limbs can be found also in animals (e.g. lobster claws, bird beaks, the tongs of certain lizards, etc.), it has maximum functionality in humans. In the case of the human hand, the various combinations of the independent motions of the phalanges allow the seizing, holding, moving and releasing of various bodies. Studies carried out to date revealed that considering the object grasping ability of a 5-finger hand as $100 \%$, a 4-finger mechanical hand has $99 \%$ of its ability, a three-finger one about $90 \%$, while a hand with two "fingers" also known as jaws merely $40 \%$ [2].

The human gripping function entails a sequence of phases, starting with selecting the method of seizing, followed by the actual gripping and control over the manipulated object. A wish or desire, whether generated internally or externally, triggers visual reactions and reactions in the brain. Consequently to such reactions the hand is positioned in the proximity of the object to be gripped, then seizes it and conducts the programmed task. Figure 1 illustrates the various phases and the links between these, as entailed by gripping [3],[4].

In robotics, gripping entails the contact between the final element (the gripper) of a robot and a body, in order to manipulate it. Grippers are those components of robot systems that facilitate the temporary contact with the manipulated object, ensuring its position and orientation during transport and assembling.

According to the classification criterion used for gripping systems taking into account their destination, grippers are deployed in either industrial applications or in research, in which latter case these being mechanical hands. The systems deployed in industrial applications are characterised by the developed force and their structural rigidity. These characteristics can be ensured by conceiving grippers with few degrees of mobility, thus facilitating system reliability and a low cost.

Corresponding Author: Prof.dr.eng. Andrea Deaconescu, Transilvania University of Brasov, Romania, 29 Eroilor Bd., Brasov, 500036, email: tdeacon@unitbv.ro 


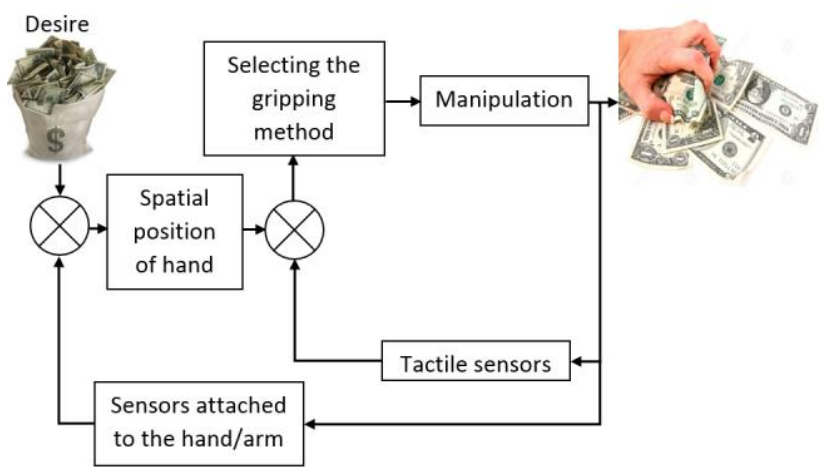

Fig 1 Command flows of a human hand gripping action and the diagram of the phases entailed by gripping

The construction of mechanical hands resembles that of the human hand and is characterised by the dexterity given by the available multiple degrees of mobility and a property known as compliance.

Many robotic applications like prostheses, medical rehabilitation devices and assembling systems require Variable Stiffness Actuators (VSAs), also known as Adjustable Compliant Actuators (ACAs).

These types of actuators offer the possibility of minimising the large forces occurring in collisions (shocks), allow safe interaction with the user and have the capacity of storing and releasing energy into passive elastic elements. Utilisation of adjustable compliance actuators ensures the adaptability of the gripping system to the concrete working situation, which in some cases differs from the one planned initially. In such a system that includes compliant actuators the presence of sensors or complex controllers is no longer required, because it is the mechanical system itself that provides the required adaptive behaviour.

Over the last years numerous efforts were made to create novel gripping systems adaptable to the environmental constraints, with a simple as possible construction and easy to control. In this context, the study presented in this paper is aimed at discussing novel, light, and eco-friendly gripping systems of bionic type. The gripping motions are generated by original, self-adaptive, bio-inspired systems, with the pneumatic muscle as the motor element.

At present worldwide many constructive solutions of pneumatic muscle actuated gripping systems are known. One of these, presented in figure 2 is called Power Gripper [5]. The design of its construction started from the way birds grasp with their beaks and its kinematics is based on Watt linkages. The system has a good developed force - to - eigenweight ratio, due to the deployment of a light motor (the pneumatic muscle).

The solutions of gripping systems proposed in this paper are of non-anthropomorphic type, and are meant for various robotic, industrial or medical applications. The method used for developing novel gripping systems is design by analogy, a powerful working tool aimed at widening the field of inspiration in design, at increasing the number of proposed constructive variants, and consequently the setting up of a data base of solutions of gripping systems that lend themselves for various applications.

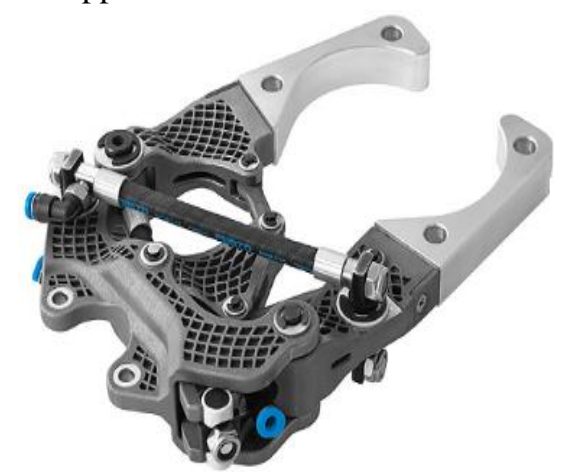

Fig 2 Pneumatic muscle actuated gripping system (Power Gripper)

\section{METHODOLOGY FOR THE DESIGN OF GRIPPING SYSTEMS}

Analogy-based design is a frequently used working method for the generation of novel products and/or technologies. Numerous scientific papers address the issue of systematic knowledge transfer from natural sciences, from biology to the field of engineering, and in this context bionic design (biomimetics) offers an enormous potential for the development of new products and technologies [6].

The functional morphology of living organisms regarded from an engineering angle represents a permanent source of inspiration for identifying innovative high-tech constructive solutions. In this respect, since the 1960s a new branch of science has been developed, namely bionics (biomimetics), combining notions from biology, mathematics, medicine, and engineering. Bionics is based on biological intuition and engineering pragmatism in order to adapt nature's projects to the necessities of the modern technical field. Nature is merely the starting point for innovations, it offers only clues as to what is useful in a mechanism. Starting from this clue, it is the engineers' task to develop and improve the analysed system. Thus bionics represents the action of systematic 
learning from nature, a different concept from "inspiring (copying) from nature" [7],[8].

Amongst the objectives of current bionic research special attention comes to the study of effector elements. Study of the effector elements and of the transmission processes of commands to these represent an essential part of bionics. In this respect land animals, birds and fishes are the inspiration source for the development of various motion generating systems.

Natural gripping systems represent a continuous source of inspiration for engineers and in time have underlain many practical applications. Thus figure 3 presents the transfer of the concept of gripping an egg by a human hand into conceiving a bio-inspired mechatronic system.

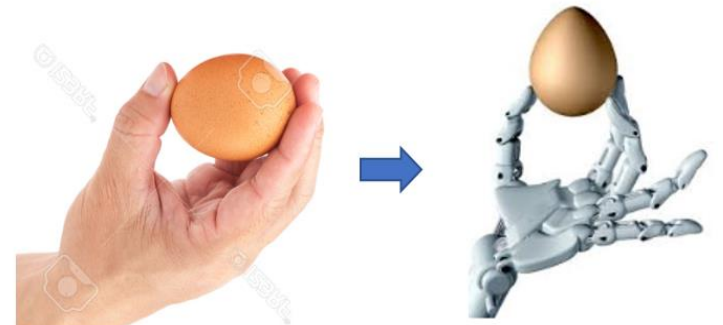

Fig 3 Transfer of a model of natural gripping to an artificial system
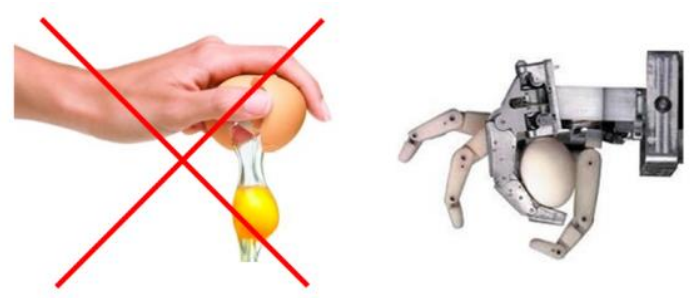

Fig 4 Applying a controlled / not controlled force to gripping the object

An artificial gripping system needs to ensure not only the seizing of the object, but its safe manipulation as well. Thus, in the case of the egg in the above example a further requirement is to control the applied force, such as to not deteriorate the object (Fig. 4). This is the case of compliant behaviour.

Starting from the above examples it follows that novel, innovative, bio-inspired solutions of gripping systems require light, flexibly structure equipment with a large useful load - to - eigenweight ratio, with integrated position adjustment and force control, offered at an affordable price. To date no gripping systems are available that meet the majority of these requirements.

\section{CONSTRUCTION OF THE GRIPPING SYSTEMS}

Figure 5 presents some of the main phases of the gripping process, namely approaching the object, initiation of contact with it and its firm gripping between the two jaws by applying an increasing force [9]. For these phases the classical profile of the jaw velocities is presented, as well as the desired and necessary shape of the curve describing the compliance of the entire system. The two graphs suggest that in the absence of contact between the jaws and the object compliance can have smaller values, what allows a better positioning precision due to higher stiffness. The curve that describes the variation of compliance has to be of concave shape, what allows a stronger increase of compliance as the moment of contact initiation between the jaws and the object is approached. Compliance has to reach its greatest values once contact has been achieved, thus ensuring safe gripping of the object without destroying it.

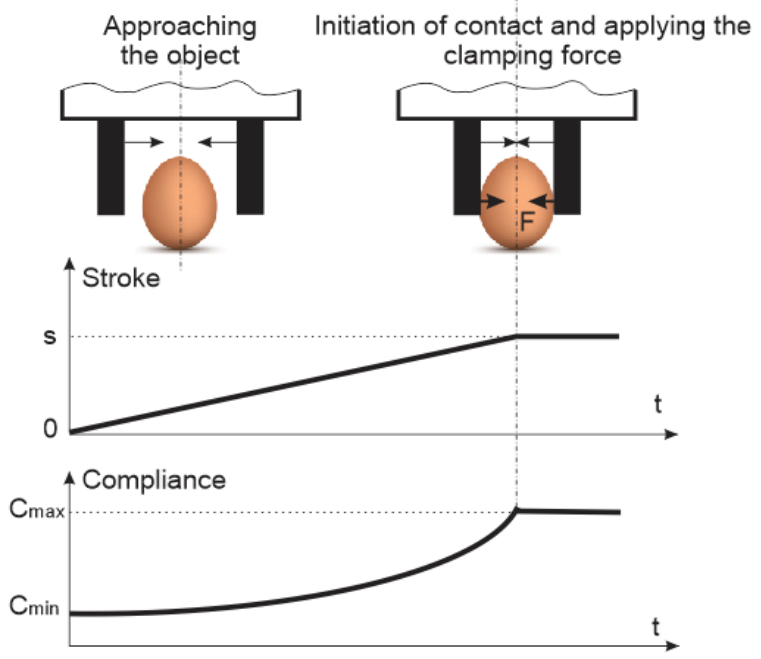

Fig 5 Variation versus time of jaw stroke and system compliance

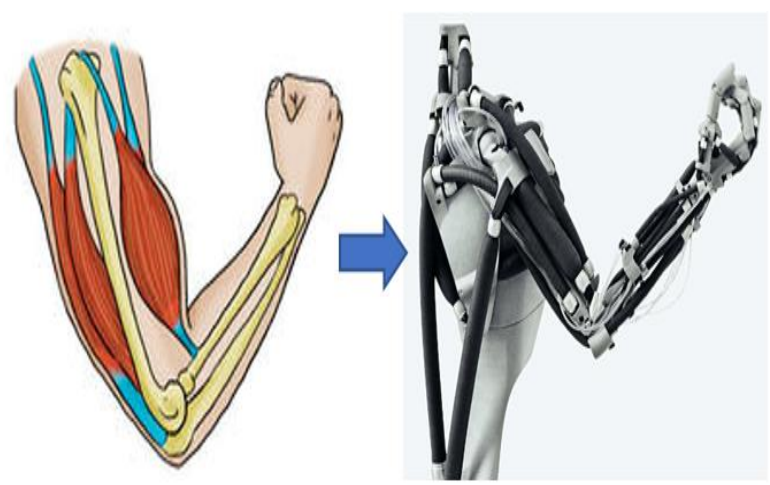

Fig 6 Pneumatic artificial muscles mimic a biological muscle

An adjustable compliance actuator like the pneumatic muscle can adapt its functional behaviour between two limits. Thus its behaviour can vary from very rigid - as required for ensuring a good positioning precision - to compliant, when the main requirement is the safety of the motion.

The pneumatic muscle copies by biomimetics the functioning of the human muscle fibre (Fig. 6), and has 
a number of characteristics like the capacity to absorb and resist shocks, low weight, low overall dimensions and mass per unit of power, and elasticity. These characteristics render pneumatic muscles optimum constructive elements for robotic applications, for mechanisms conducting orientation as well as gripping. Further presented and discussed are four variants of gripping systems using the pneumatic muscle as actuator and a power transmission mechanism consisting of gears.

The input data below were the specifications used for dimensioning the pneumatic muscle actuated gripping systems:

- mass of the object: $\mathrm{m}=0.7 \mathrm{~kg}$;

- acceleration of the motion carried out by the gripper + object system: $\mathrm{a}=5 \mathrm{~m} / \mathrm{s}^{2}$;

- gravitational acceleration: $\mathrm{g}=9.81 \mathrm{~m} / \mathrm{s}^{2}$;

- emergency stopping acceleration: $\mathrm{aS}=10 \mathrm{~m} / \mathrm{s}^{2}$;

- friction coefficient: $\mu=0.2$;

- safety coefficient: $\mathrm{S}=2.5$.

For these input data the maximum force required for gripping in the different phases of manipulation are calculated by:

$$
F_{\text {jaw }}=\frac{m \cdot\left(g+a_{S}\right) \cdot S}{\mu \cdot n}=\frac{0.7 \cdot(9.81+10) \cdot 2.5}{0.2 \cdot 2}=86.67 \mathrm{~N}
$$

As all proposed gripping systems have two jaws, the force to be generated by the pneumatic muscle will be double, of minimum 173.34 N (without considering the efficiencies of the linkages).

In view of the imposed requirements as to operation and overall dimensions, the proposed models discussed in this paper deploy the smallest pneumatic muscle manufactured by the German company Festo (MAS-1045N-AA-MC-O-ER-EG), with the following characteristics: muscle diameter $10 \mathrm{~mm}$; length of the active part $45 \mathrm{~mm}$; maximum working pressure: 6 bar.

Figure 7 presents the graph that describes the evolution of the force developed by the selected pneumatic muscle versus charging pressure and stroke. The hachured rectangle highlights the optimum deployment area of the pneumatic muscle, so that for a maximum stroke of the jaws of $4 \mathrm{~mm}$, the developed force exceeds $173.34 \mathrm{~N}$.

Figures 8 to 11 show the diagrams of principle of the four variants of gripping systems.

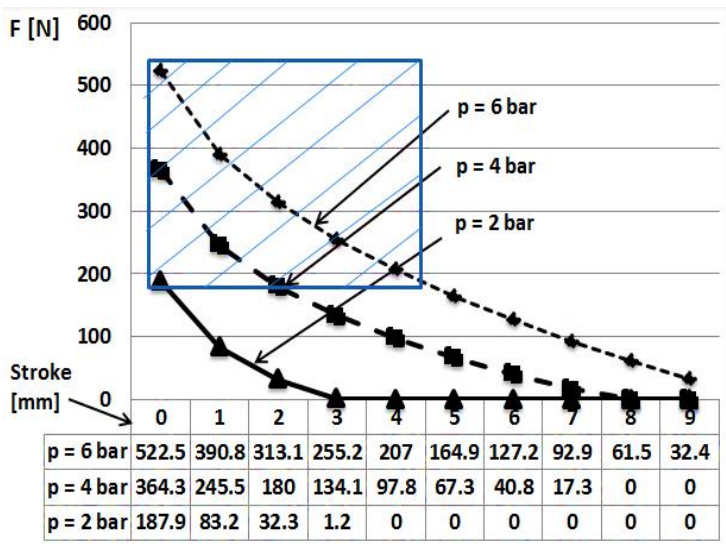

Fig 7 Variation of the developed force versus the charging pressure and stroke

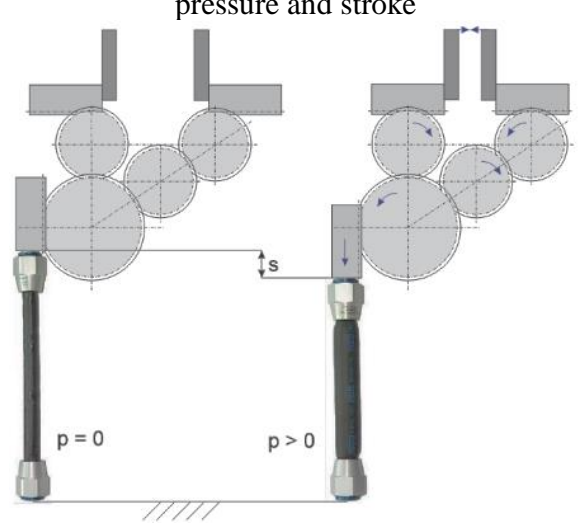

Fig 8 Variant 1: Parallel asymmetrical gripping system with two mobile jaws

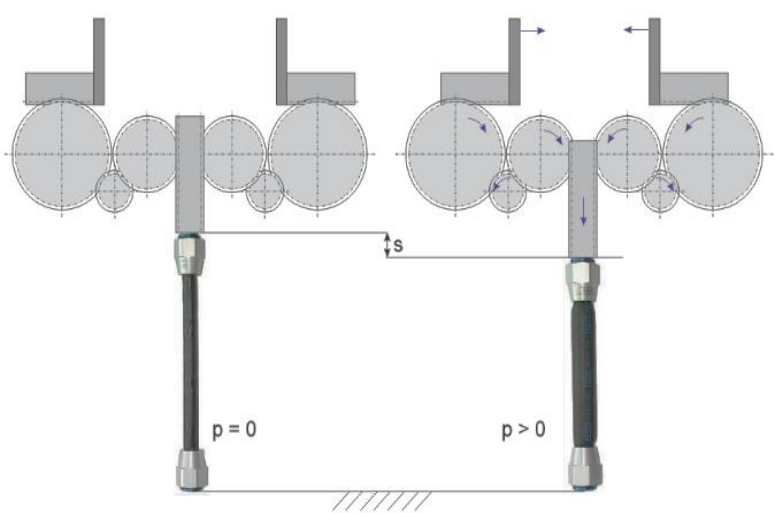

Fig 9 Variant 2: Parallel symmetrical gripping system with two mobile jaws 


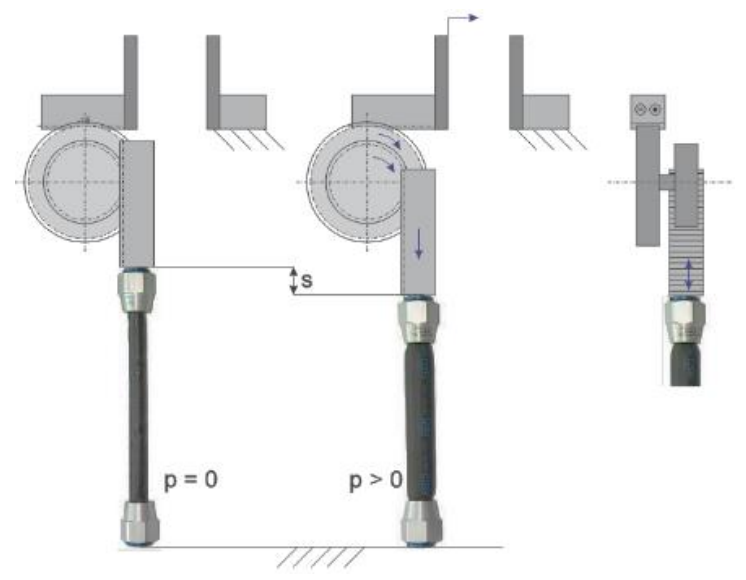

Fig 10 Variant 3: Parallel asymmetrical gripping system with a mobile jaw and a fixed jaw

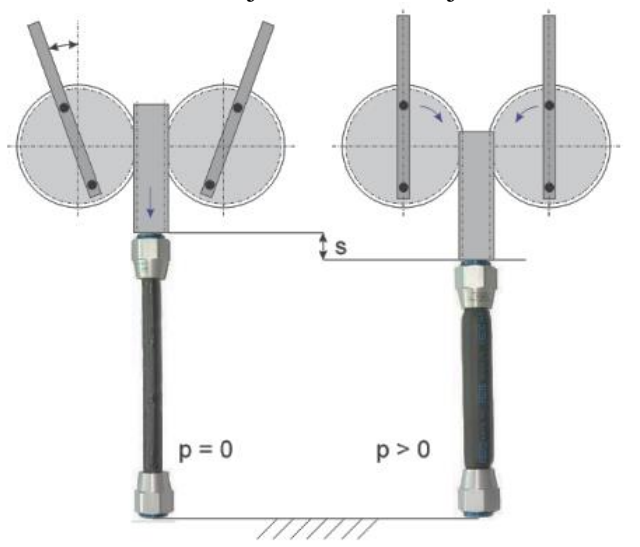

Fig 11 Variant 4: Angular gripping system with two mobile jaws

All four variants of proposed gripping systems benefit from compliant behaviour, what ensures the safe manipulation of fragile objects, preventing their deformation or destruction. The deployment of a pneumatic muscle as an adjustable compliant actuator allows deviations from the position of equilibrium, their magnitude depending on the level of the exterior forces the muscle is subjected to.

\section{ACTUATION OF THE GRIPPER SYSTEMS}

Figure 12 presents the actuation diagram of the gripping system. The contraction of the pneumatic muscle is controlled by means of a 3/2-way fast-switching normally closed solenoid valve, manufactured by Festo. The evolution of pressure within the pneumatic muscle versus time was studied for the proposed gripping system for object seizing and releasing operations (Fig. 13). Measurements were carried out by means of an analogous pressure gauge connected to the computer via a digital/analogous EasyPort measurement interface. The role of the FluidLab®-P V1.0 programme is to plot the evolution versus time of the analysed parameters.
The motion onset of the pneumatic muscle free end is characterised, as expected also following analysis of the functional model, by the occurrence of a short pressure impulse of $0.086 \mathrm{MPa}$ amplitude and $0.019 \mathrm{~s}$ duration. After the imposed pressure has been reached, its value records only small deviations, the maximum amplitude of such fluctuations being $0.0029 \mathrm{MPa}$, of no relevance to the quality of gripping.

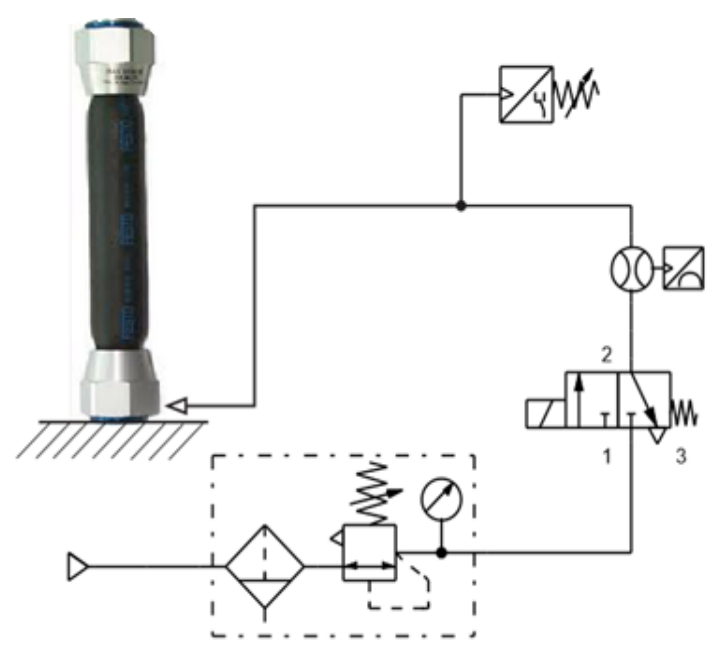

Fig 12 Actuation diagram of the gripping system

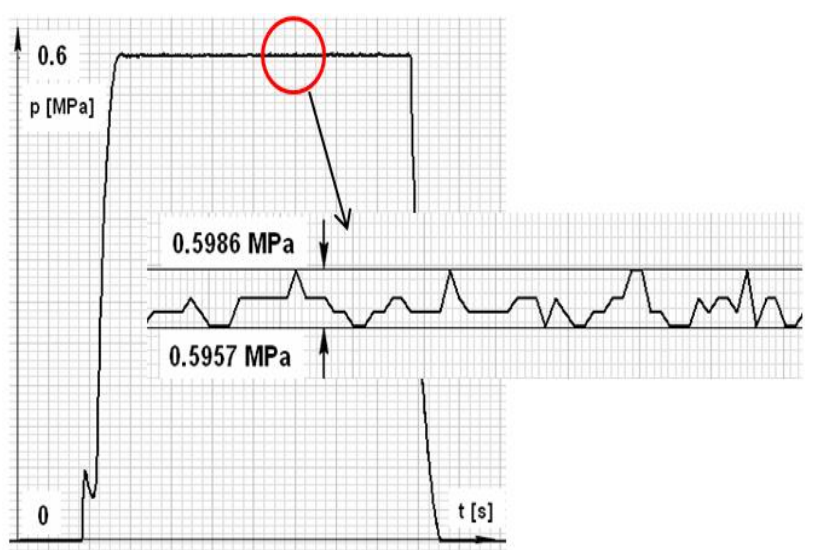

Fig 13 Evolution of the pneumatic muscle feeding pressure 


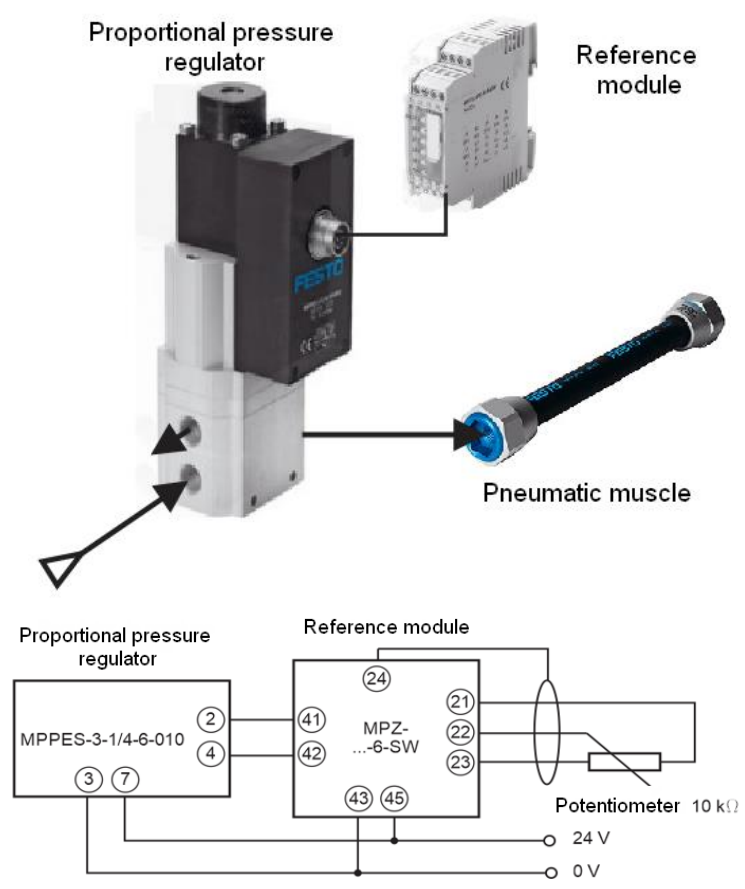

Fig 14 Actuation diagram of the gripper with a proportional pressure regulator

In the case where the 3/2-way fast-switching normally closed solenoid valve is replaced by a proportional pressure regulator of MPPES-3-1/4-6-010 type (Fig. 14), manufactured by Festo, the output pressure will be proportional to a pre-set value of the input quantity, which has the form of a voltage type signal. The proportional pressure regulator is controlled by a reference module MPZ-1-24DC-SGH-6-SW, also made by Festo. By means of rotational potentiometers the reference module can generate up to six different values of the reference voltage, which are transmitted in form of signals to the proportional regulator. If none of these reference values is used, the signal transmitted to the pressure regulator is a voltage adjustable via an external potentiometer. By means of this potentiometer the output pressure of the regulator is controlled, and thus the stroke carried out by the pneumatic muscle.

\section{CONCLUSION}

The paper has presented and discussed several innovative constructive solutions of non- anthropomorphic gripping systems conceived to ensure safe gripping and manipulation of fragile objects. The proposed variants are based on the utilisation of a linear pneumatic muscle as motion generating element and on the transmission of motion to the jaws by means of gears and racks. The main advantages of the proposed gripping system are small weight and low cost, as well as easy mounting on the various existing robots.

The idea of using pneumatic muscles as actuators was taken from nature by biomimetics, based on the functioning of the human muscle fibre.

Using a linear pneumatic muscle as the actuator of the gripping system ensures a light construction of particularly compliant behaviour that corresponds to the safety requirements for interaction with humans.

\section{REFERENCES}

[1] Dicţionarul explicativ al limbii române [Romanian Language Dictionary]. http://www.dex-online.ro/

[2] Stăncescu, C. 2009. Theoretical and experimental study of gripping systems with jaws used in industrial robots, (in Romanian) $\mathrm{PhD}$ Thesis, Universitatea Transilvania din Braşov

[3] Nof, S. (editor) 1985. Handbook of Industrial Robotics (John Wiley and Sons Inc. New York)

[4] Țârliman, D. 2014. Research concerning the gripping systems of industrial robots actuated by pneumatic muscles (in Romanian), PhD Thesis, Universitatea Transilvania din Braşov

[5] Festo. PowerGripper Research project for the development of new gripping systems http://www.festo.com/cms/en_corp/12728.htm

[6] Christensen, B. T., Schunn, C. D. 2007. The Relationship of Analogical Distance to Analogical Function and Pre-inventive Structure: The Case of Engineering Design, Memory \& Cognition, 35(1) 29-38

[7] Dieter, G. E. 2000. Engineering Design: A Material and processing Approach (McGraw-Hill)

[8] Ulrich, K. T., Eppinger, S. D. 2004. Product Design and Development (Boston: McGraw-Hill/Irwin)

[9] Deaconescu, T., Deaconescu, A. 2017. Pneumatic Muscle Actuated Adjustable Compliant Gripping System for Assembly Operations Strojniški vestnik - Journal of Mechanical Engineering 63/4 225-234, DOI:10.5545/sv-jme.2016.4239 